\title{
Guest-editorial
}

\section{Special Issue on VIII Brazilian Symposium on Neural Networks}

These are two extended versions of papers selected among the best evaluated accepted papers for the VIII Brazilian Symposium on Neural Networks. The Symposium accepts papers on different topics on Computational Intelligence, in particular, the event is interested in neural networks, evolutionary algorithms, and fuzzy logic models and applications. SBRN has an international Program Committee, with well known international researchers. In the last editions, the SBRN proceedings were published by IEEE Computer Society.

São Luis, Maranhão, Brazil, was the venue of the SBRN'2004. The sponsors were the Brazilian Computer Society (SBC) and the SIG/INNS/Brazil Special Interest Group of the International Neural Networks Society in Brazil. SBRN'04 had 154 papers accepted out of 329 submissions from several countries. The papers described below are extended versions among the best conference papers.

The paper called the interpretation of feedforward neural networks for secondary structure prediction using Sugeno fuzzy rules, by Cechin, and Battistella, involves rule extraction process applied to multilayer neural networks to predict protein backbone dihedral angles based on the physical-chemical attributes of the amino acids. The authors analyzed the knowledge learned by the neural network as a fuzzy inference system of Sugeno fuzzy rules. The proposed rules have the nice feature of being linear in relation with the input parameters.

Da Silva, Goedtel, and Flauzino, in their paper a modified Hopfield architecture applied in dynamic programming problems and bipartite graph optimization, introduce a modified version of Hopfield architecture in which the equilibrium points represent the solution of dynamic programming problems and bipartite graph optimization. The valid-space technique was employed to determined the Hopfield parameters in order to deal only with viable solutions. The model was tested and compared with other methods.

Canuto, Bezerra, Abreu, and Campos present an agent-based system for knowledge discovery from database. The paper, called investigating the use of a multi-agent system for knowledge discovery in databases, describes a system formed by knowledge discovery agents responsible for data preparation and data mining. Each agent has a different data mining method, hence, they may answer differently a particular question. Finally, the potential distinct answers are processed through a negotiation method to provide a commonly agreed output.

A new hybrid heuristic for driver scheduling, authored by Mauri and Lorena, describes a hybrid model, mixing Population Training Algorithm (PTA) and linear programming (LP), to generate schedules for drivers of public transport system. The problem is divided into subproblems with PTA generating low cost and good covering of the tasks and LP solving a set of partitioning problem formed by the options previously provided. PTA heuristically finds promising solutions whereas LP finds the final solutions. The computational results are compared with a Simulated Annealing approach using randomly formed instances based on real problems. 
The editors thank all the authors for their contribution to this issue, the referees for their reviews to improve the quality of the papers. Thanks also to the International Journal of Hybrid Intelligent Systems (IJHIS), in particular to Prof. Lakhmi Jain and Prof. Ajith Abraham for their support for this issue. Federal University of Pernambuco E-mail: aluizioa@cin.ufpe.br

André C. P. L. F. de Carvalho University of São Paulo E-mail: andre@icmc.usp.br

Antônio P. Braga Federal University of Minas Gerais E-mail: apbraga@cpdee.ufmg.br 